\section{Serum Levels of HMGB1 in Postmenopausal Patients with Rheumatoid Arthritis: Associations with Proinflammatory Cytokines, Acute-phase Reactants, and Clinical Disease Characteristics}

\section{To the Editor:}

High mobility group box 1 (HMGB1) chromosomal protein, an endogenous molecule with multiple intra- and extracellular effects, is also defined as a novel proinflammatory cytokine, proved to contribute to the pathogenesis of several rheumatic diseases ${ }^{1}$. Evidence grows showing that HMGB1 plays a role as an important endogenous mediator of arthritis. Significantly elevated levels of HMGB1 are reported in the sera and synovial fluid of patients with rheumatoid arthritis (RA) as compared to patients with osteoarthritis ${ }^{2}$. Levels of HMGB1 are increased in the synovial tissues of animals and humans with joint inflammation ${ }^{3}$. HMGB1 mediates the development of arthritis when applied into the joints of naive mice $^{4}$ and treatment strategies directed against HMGB1 attenuate progression in collagen-induced arthritis ${ }^{1}$. In RA, characterized by chronic autoimmune destructive joint inflammation, extensive production of proinflammatory cytokines is known to mediate tissue damage.

HMGB1, either released actively from myeloid and dendritic cells or passively during local cell death in the joint, could sustain chronic synovitis by several mechanisms including an augmentation of production of several proinflammatory mediators such as interleukin 1 (IL-1), tumor necrosis factor- $\alpha$ (TNF- $\alpha$ ), and IL-6, all known to be important in the pathogenesis of arthritis ${ }^{5}$.

In a cross-sectional study, we investigated the relationship between concentrations of HMGB1 and proinflammatory cytokines and disease characteristics in a cohort ${ }^{6}$ of 81 postmenopausal patients with RA. The characteristics of patients are summarized in Table 1. Laboratory and clinical values were recorded and radiographs of the hands, wrists, and forefeet were evaluated according to the Larsen method ${ }^{6}$.

Table 1. Demographic and disease-related characteristics of postmenopausal women with rheumatoid arthritis $(n=81)$. Values are medians (25th-75th percentiles). The number of patients/total number (\%) is shown. Spearman rank correlation coefficient and $\mathrm{p}$ value are indicated for correlations between HMGB1 and disease-related measures.

Characteristics Median Value $\begin{gathered}\text { Correlation } \\ \text { Coefficient (rho) }\end{gathered}$

\begin{tabular}{lccc}
\hline Serum HMGB1, pg/ml & $887(0-1592)$ & & \\
Age, yrs & $58(53-63)$ & & \\
Disease duration, yrs & $14(6.8-22.2)$ & 0.32 & 0.006 \\
DMARD & $66 / 81(81 \%)$ & & \\
Glucocorticoids at present & $17 / 81(21 \%)$ & & \\
Positive rheumatoid factor & $68 / 81(84 \%)$ & & \\
Erosive disease & $71 / 79(90 \%)$ & & \\
Larsen score & $1.18(0.54-2.12)$ & 0.22 & 0.06 \\
Disease Activity Score 28 joints & $5.3(4.7-5.9)$ & 0.20 & 0.09 \\
Swollen joint count & $9(6-13)$ & 0.24 & 0.04 \\
Tender joint count & $10(5-15)$ & 0.22 & 0.07 \\
C-reactive protein, mg/1 & $9(6-19)$ & 0.26 & 0.02 \\
Orosomucoid, g/l & $1.1(0.9-1.4)$ & 0.32 & 0.004 \\
Health Assessment Questionnaire & & & \\
$\quad$ score & $1.0(0.38-1.53)$ & 0.23 & 0.04 \\
IL-6, pg/ml & $8.4(2.1-35.0)$ & 0.28 & 0.01 \\
Soluble IL-6 receptor, pg/ml & $749(608-926)$ & 0.26 & 0.02 \\
IL-1ß, pg/ml & $0.1(0.1-0.3)$ & -0.03 & 0.81 \\
IL-1 receptor antagonist, pg/ml & $406(288-527)$ & 0.21 & 0.06 \\
TNF- $\alpha$, pg/ml & $3.4(2.5-4.9)$ & -0.11 & 0.26 \\
\hline
\end{tabular}

DMARD: disease-modifying antirheumatic drug; IL-6: interleukin 6; TNF- $\alpha$ : tumor necrosis factor- $\alpha$.
The ELISA method was used to measure serum levels of TNF- $\alpha$, IL-1ß, IL-6, IL-1RA, sIL-6R (R\&D Systems), and HMGB1 (Shino-Test Corp., Kanagawa, Japan). Amounts of HMGB1 were also determined in parallel with Western blots in 20 patients ${ }^{7}$. For statistical analysis nonparametric tests were employed due to unequal data distribution. Data are presented as medians (25th-75th percentiles).

The median HMGB1 concentration was 887 (range 0-1592) pg/ml, with $30 \%$ of patients displaying undetectable levels. HMGB1 concentrations were significantly positively correlated with disease duration (Figure 1A). No significant differences were observed between patients taking ( $\mathrm{n}=$ 66 ; median 962, range $0-1571)$ or not taking $(\mathrm{n}=15$; median 365 , range 0-1562) disease-modifying antirheumatic drugs. Women treated with oral corticosteroids ( $\mathrm{n}=17$; median 1015, range 324-1984) had HMGB1 levels comparable to patients not receiving corticosteroids $(n=64$; median 869 , range $0-1523$ ).

HMGB1 levels correlated significantly with levels of IL-6 and sIL-6R, but no associations were found with TNF- $\alpha$ or IL-1ß (Table 1). Only 48\% of the patients had detectable serum levels of IL-1ß. HMGB1 was significantly associated with swollen joint count and acute-phase reactants, C-reactive protein, and orosomucoid. Patients with erosive disease had a tendency to have higher HMGB1 concentrations (median 961, range $11-1726 \mathrm{pg} / \mathrm{ml} ; \mathrm{p}=0.09$ ) than patients without erosions (median 0 , range 0-1078). HMGB1 levels were significantly associated with Health Assessment Questionnaire (HAQ) score. HMGB1 levels by Western blot were generally higher (median 4680, range $0-35,375 \mathrm{pg} / \mathrm{ml}$ ) than those detected by ELISA (median 1877, range 204-3772 pg/ml; Figure 1B).

We observed a modest correlation between HMGB1 levels obtained by ELISA and those from Western blots, in accord with results described by Urbonaviciute, et $a l^{7}$. Considering the potential human serum factors including HMGB1-specific antibodies masking $\mathrm{HMGB}^{7}$, it is plausible that the total circulating HMGB1 levels detected by Western blotting are higher than those detectable by ELISA. Possibly, HMGB1 ELISA detects predominantly free, biologically active HMGB1, which might be responsible for induction of the inflammatory response.

Although TNF- $\alpha$ and IL-1ß, pivotal cytokines in the pathogenesis of arthritis, have the ability to induce the release of HMGB1 from myeloid and dendritic cells, we found no correlation between systemic HMGB1 and TNF- $\alpha /$ IL-1ß levels in our study population. Recent research suggests that HMGB1 signaling and activity might be independent of TNF during joint inflammation ${ }^{8,9}$. Systemic IL-1ß levels are low and were detectable in only $48 \%$ of patients in our RA cohort, which might explain the absence of significant associations between HMGB1 and IL-1ß levels.

The presence of chronic synovitis in RA generates the basis for release of HMGB1 and could thereby perpetuate local as well as systemic inflammation by enhancing the expression of proinflammatory cytokines ${ }^{1,5}$. Studies show that HMGB1 is also a bone-active cytokine released from osteoclasts, osteoblasts, and dying osteocytes ${ }^{10}$. It can induce the release from bone marrow stromal cells of IL-6, one of the most important cytokines in osteoclastogenesis, and therefore contribute to inflammatory bone $\operatorname{loss}^{10}$. We observed a significant correlation between HMGB1 levels and IL-6 as well as sIL-6R in our RA cohort. Patients with erosive disease had a tendency to higher levels of HMGB1, suggesting that HMGB1 might be important in bone degradation. We previously demonstrated in a mouse model that HMGB1 induces long-lasting inflammation and cartilage erosions and pannus formation in the singularly exposed joint ${ }^{4}$. In addition, HMGB1 levels were positively associated with disease duration, but not with the age of patients, further supporting its involvement in chronic inflammation.

Levels of HMGB1 were significantly associated with acute-phase markers and also with swollen joint count and HAQ score, underlining its role as an inflammatory mediator. These associations between variables were considered biologically relevant and clinically meaningful and pointed in the same direction despite, statistically, a relatively small study population; the statistical analysis was not corrected for multiple comparisons and the results should thus be interpreted with care. 

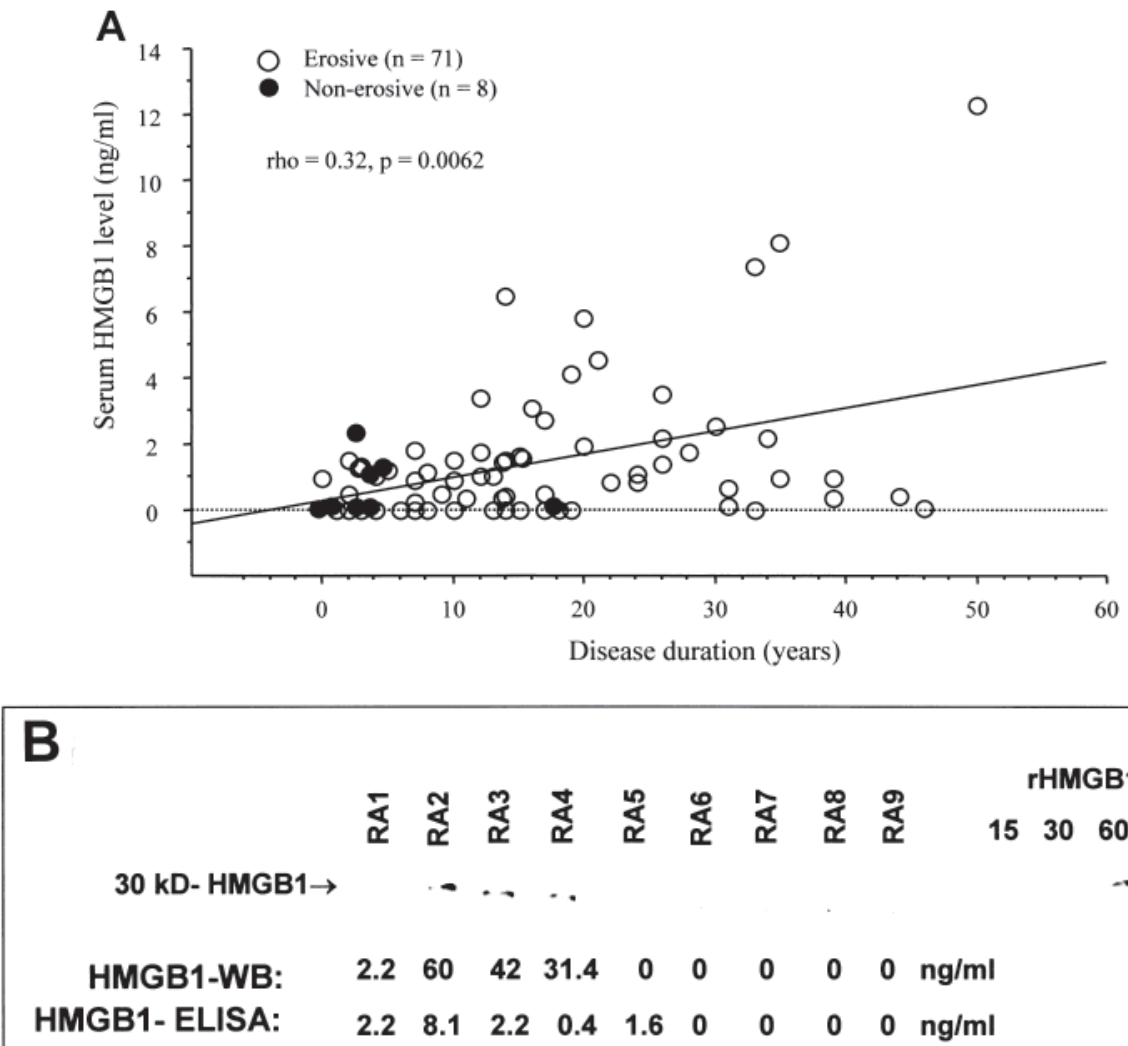

Figure 1. Serum levels of HMGB1 detected by ELISA in postmenopausal patients with RA are significantly associated with disease duration (A; regression line and correlation coefficient are shown) and display only a modest correlation with HMGB1 concentrations on Western blot (B). Data from one experiment illustrating HMGB1 levels detected with ELISA versus Western blot are shown (B).

Our results suggest that serum HMGB1 levels in postmenopausal women with RA could serve as a useful marker of inflammatory activity in these patients. Based on our own and others' results, we hypothesize that HMGB1 might play a role in inflammation-related bone loss and thus constitute a potential therapeutic target. However, since reliable quantification of HMGB1 remains an unresolved issue, all results obtained by ELISA in human serum samples should be interpreted with care. Future prospective studies are needed to determine whether HMGB1 is a potential marker of disease activity and severity and/or a predictor of outcome in RA patients in general.

RILLE PULLERITS, MD, PhD, Department of Rheumatology and Inflammation Research, University of Gothenburg, Gothenburg; VILMA URBONAVICIUTE, MD; REINHARD E. VOLL, MD, Prof., Nikolaus Fiebiger Center of Molecular Medicine, University Hospital Erlangen, University of Erlangen-Nuremberg, Erlangen, Germany; HELENA FORSBLAD-D'ELIA, MD, PhD; HANS CARLSTEN, MD, Prof., Department of Rheumatology and Inflammation Research, University of Gothenburg, Gothenburg, Sweden.

Address correspondence to Dr. R. Pullerits, The Sahlgrenska Academy, University of Gothenburg, Department of Rheumatology and Inflammation Research, Guldhedsgatan 10A, 41346, Gothenburg, Sweden. E-mail: rille.pullerits@rheuma.gu.se

Supported by grants from the Gothenburg Medical Society, the Swedish Medical Society, the Swedish Association Against Rheumatism, the
Gothenburg Association Against Rheumatism, the King Gustaf V Foundation, the Swedish Medical Research Council, the Nanna Svartz Foundation, Rune and Ulla Amlövs Foundation, and the University of Gothenburg.

\section{REFERENCES}

1. Pisetsky DS, Erlandsson-Harris H, Andersson U. High-mobility group box protein 1 (HMGB1): an alarmin mediating the pathogenesis of rheumatic disease. Arthritis Res Ther 2008;10:209.

2. Ulloa L, Batliwalla FM, Andersson U, Gregersen PK, Tracey KJ. High mobility group box chromosomal protein 1 as a nuclear protein, cytokine, and potential therapeutic target in arthritis. Arthritis Rheum 2003;48:876-81.

3. Taniguchi N, Kawahara K, Yone K, Hashiguchi T, Yamakuchi M, Goto $\mathrm{M}$, et al. High mobility group box chromosomal protein 1 plays a role in the pathogenesis of rheumatoid arthritis as a novel cytokine. Arthritis Rheum 2003;48:971-81.

4. Pullerits R, Jonsson IM, Verdrengh M, Bokarewa M, Andersson U, Erlandsson-Harris $\mathrm{H}$, et al. High mobility group box chromosomal protein 1, a DNA binding cytokine, induces arthritis. Arthritis Rheum 2003;48:1693-700.

5. Jiang W, Pisetsky DS. Mechanisms of disease: the role of high-mobility group protein 1 in the pathogenesis of inflammatory arthritis. Nat Clin Pract Rheumatol 2007;3:52-8.

6. Forsblad D'Elia H, Larsen A, Waltbrand E, Kvist G, Mellstrom D, Saxne T, et al. Radiographic joint destruction in postmenopausal 
rheumatoid arthritis is strongly associated with generalised osteoporosis. Ann Rheum Dis 2003;62:617-23.

7. Urbonaviciute V, Furnrohr BG, Weber C, Haslbeck M, Wilhelm S, Herrmann M, et al. Factors masking HMGB1 in human serum and plasma. J Leukoc Biol 2007;81:67-74.

8. Pullerits R, Jonsson IM, Kollias G, Tarkowski A. Induction of arthritis by high mobility group box chromosomal protein 1 is independent of tumour necrosis factor signalling. Arthritis Res Ther 2008;10:R72.
9. Sundberg E, Grundtman C, Af Klint E, Lindberg J, Ernestam S, Ulfgren AK, et al. Systemic TNF blockade does not modulate synovial expression of the pro-inflammatory mediator HMGB1 in rheumatoid arthritis patients - a prospective clinical study. Arthritis Res Ther 2008;10:R33.

10. Yang J, Shah R, Robling AG, Templeton E, Yang H, Tracey KJ, et al. HMGB1 is a bone-active cytokine. J Cell Physiol 2008;214:730-9.

J Rheumatol 2011;38:7; doi:10.3899/jrheum.110091 